\title{
A comparison of the effects of protein supplementation and resistance exercise on UK South Asian and white European middle-aged adults
}

\author{
M. Shah ${ }^{1}$ and R. Mackenzie ${ }^{2}$ \\ ${ }^{1}$ Faculty of Science, Engineering and Computing, Kingston University, Penrhyn Road, Kingston upon Thames, KT1 \\ $2 E E$ and \\ ${ }^{2}$ Department of Life Sciences, Roehampton University, Whitelands College, Holybourne Ave, Roehampton, London
}

SW15 4JD

The combination of an ageing population and an age-related decline in musculoskeletal health with increased adiposity and sarcopenia, represents a major public health concern ${ }^{(1)}$. This decline in musculoskeletal health is associated with a higher risk of cardiometabolic diseases, with a reduced quality of life and associated health care costs ${ }^{(2)}$.

South Asians are a high-risk group for cardiometabolic diseases, due to ethnic differences in body composition. Thus, cardiometabolic diseases that are often associated with age-related changes in body composition, often occur much earlier in life in South Asians (3).

For adults $\geq 65 \mathrm{y}$, a combination of resistance exercise together with protein supplementation is considered the optimal intervention for improvements in muscle protein synthesis, muscle strength, function and a reduction in morbidity and mortality, as well as overall quality of life ${ }^{(4)}$. Very few studies examining the effects of protein supplementation and resistance exercise on middle-aged adults have been conducted, with most only involving male participants, and no studies found comparing the effects between ethnic groups. The objective of this study was to compare the effects of protein supplementation and resistance exercise between UK South Asian and white European middle-aged adults.

20 healthy adults (45-64y, BMI 18-29.9kg/m²) of South Asian $(\mathrm{n}=5$ women; $\mathrm{n}=5$ men) and white European $(\mathrm{n}=7$ women; $\mathrm{n}=3$ men) origin, were recruited for the 2 -week study. Participants received 2 daily doses $(1 \mathrm{dose}=0.4 \mathrm{~g} / \mathrm{kg} / \mathrm{day})$ of a whey protein supplement, consumed at breakfast and lunch, for the study duration. Resistance exercise involved 3 days per week of 3 sets of 10 repetitions of gym-based exercise, corresponding to $70 \%$ of one repetition of maximum strength (1RM). Exercises included: leg press, bench press, knee extension and flexion, shoulder press, lat pull-down, biceps curl and triceps extension. Strength tests for upper- and lowerbody strength were based on the 1RM for bench- and leg-press respectively. Anthropometric measures included: waist, hip, thigh and biceps circumferences. All tests and measures were conducted at baseline and after completion of the 2-week programme.

From baseline to week 2 , significant increases in strength $(\mathrm{P}<0.05)$ were observed in both groups, with no significant differences between groups. Waist circumference declined in both groups but was only significant in the white European group $($ mean difference $=$ $2.1 \mathrm{~cm}, \mathrm{SE}=0.64, \mathrm{P}=0.006$ ).

Resistance training and protein supplementation significantly increased strength in both South Asian and white European middle-aged adults. Waist circumference also significantly declined in white Europeans, but not in the South Asian group, indicating that South Asians may be more resistant to reductions in abdominal adiposity. Further longer-term research is required to verify these findings with additional measures to assess body composition alterations and effects on cardiometabolic disease markers.

1. WHO (2018) [Available at: www.who.int/news-room/fact-sheets/detail/ageing-and-health]

2. Dennison EM, Sayer AA \& Cooper C (2017) Nat Rev Rheumatol 13, 340

3. Gholap N, Davies M, Patel, K et al. (2011) Prim Care Diabetes 5, 45-56.

4. Morton RW, Murphy KT, McKellar SR et al. (2018) Br J Sports Med 52, 376-384. 